\title{
Inpatient and outpatient treatment patterns of cancer-associated thrombosis in the United States
}

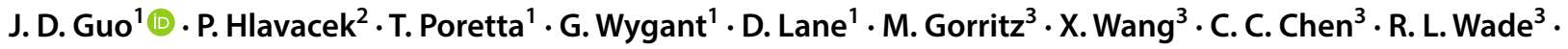 \\ X. Pan ${ }^{1} \cdot$ J. Rajpura ${ }^{1} \cdot$ B. Stwalley ${ }^{1} \cdot$ L. Rosenblatt ${ }^{1}$
}

Published online: 18 January 2020

(c) The Author(s) 2020

\begin{abstract}
Low molecular weight heparins (LMWHs) and direct oral anticoagulants (DOACs) are among the recommended treatment options for cancer-associated thrombosis (CAT) in the 2019 National Comprehensive Care Network guidelines. Little is known about the current utilization of DOACs in CAT patients, particularly on the inpatient to outpatient therapy transition. This study assessed real-world treatment patterns of CAT in hospital/ED in adult cancer patients ( $\geq 18$ years) diagnosed with CAT during a hospital visit in IQVIA's Hospital Charge Data Master database between July 1, 2015 and April 30, 2018, and followed their outpatient medical and pharmacy claims to evaluate the initial inpatient/ED and outpatient anticoagulants received within 3 months post-discharge. Results showed that LMWH and unfractionated heparin (UFH) were the most common initial inpatient/ED CAT treatments (35.2\% and 27.4\%, respectively), followed by DOACs (9.6\%); $20.8 \%$ of patients received no anticoagulants. Most DOAC patients remained on DOACs from inpatient/ED to outpatient settings (71.4\%), while $24.1 \%, 43.5 \%$, and $0.1 \%$ of patients treated with LMWH, warfarin, or UFH respectively, remained on the same therapy after discharge. In addition, DOACs were the most common initial post-discharge outpatient therapy. Outpatient treatment persistence and adherence appeared higher in patients using DOACs or warfarin versus LMWH or UFH. This study shows that DOACs are used as an inpatient/ED treatment option for CAT, and are associated with less post-discharge treatment switching and higher persistence and adherence. Further research generating real-world evidence on the role of DOACs to help inform the complex CAT clinical treatment decisions is warranted.
\end{abstract}

Keywords Cancer · Direct oral anticoagulants $\cdot$ Low molecular weight heparin · Warfarin · Venous thromboembolism · Cancer associated thrombosis

\section{Highlights}

Electronic supplementary material The online version of this article (https://doi.org/10.1007/s11239-019-02032-3) contains supplementary material, which is available to authorized users.

\section{J. D. Guo}

Jennifer.Guo@bms.com

1 Bristol-Myers Squibb, 3401 Princeton Pike, Lawrence Township, Lawrenceville, NJ 08648, USA

2 Pfizer, Inc., New York, NY, USA

3 IQVIA Inc, Plymouth Meeting, PA, USA
- LMWH and UFH were the most common initial anticoagulants used to treat CAT during an inpatient or ED visit, while DOACs were the most common initial treatments observed after discharge.

- Patients who initiated anticoagulant therapy using DOACs during an inpatient or ED visit were likely to remain on DOACs after discharge, while patients who initiated therapy using other anticoagulants were more likely to switch therapy classes after discharge.

- Patients treated with DOACs or warfarin in the outpatient setting had better treatment persistence and higher adherence than patients treated with LMWH and UFH.

- Additional real-world studies are warranted to compare clinical outcomes, such as recurrent VTE and major 
bleeding, which will help guide clinical decisions in this area.

\section{Introduction}

Cancer-associated thrombosis (CAT), also known as cancerassociated venous thromboembolism (VTE) [1-3], is the second most common cause of death in cancer patients [4]. Common forms of VTE are deep vein thrombosis (DVT) and pulmonary embolism (PE) [5, 6], which are more likely to occur in cancer patients than in the general population [4]. Risk of developing VTE in cancer patients is four to seven times higher than in patients without cancer $[2,7]$. Risk factors for CAT include chemotherapy treatment, certain hormone therapies, surgical interventions, immobilization, and cancer types $[4,8,9]$.

Complications like increased risk of recurrent VTE makes the management of CAT complex [10]. Several anticoagulant treatment options are indicated for CAT. Parenteral therapies include low-molecular-weight heparins (LMWH) such as dalteparin, and unfractionated heparin (UFH). Oral therapy options include vitamin $\mathrm{K}$ antagonists (e.g., warfarin), and direct-acting oral anticoagulants (DOACs: apixaban, betrixaban, dabigatran, edoxaban and rivaroxaban).

Recent clinical trial data of DOACs for the treatment of CAT have been promising, showing lower rates of VTE recurrence in patients treated with DOACs compared to LMWHs [11, 12]. The ADAM-VTE [11] and SELECT-D [12] trials of apixaban and rivaroxaban, respectively, versus dalteparin in CAT patients showed that DOACs had significantly lower rates of VTE recurrence than the LMWH comparator.

In light of favorable clinical trial results, treatment guidelines for CAT are evolving; the 2019 National Comprehensive Cancer Network (NCCN) guidelines for the treatment of CAT was the first to recommend DOACs. Per the 2019 NCCN guidelines, recommended CAT treatments include LMWH, UFH, or rivaroxaban as monotherapy options; apixaban is recommended as an alternative for patients who refuse or have compelling reasons to avoid LMWH [13].

There is little real-world data on anticoagulant treatment patterns in CAT patients. Most studies report treatments observed either in the hospital or outpatient setting $[14,15]$. The transition of anticoagulant treatment from the inpatient to outpatient setting was reported in one abstract [16]. The objectives of this study were to (1) describe the demographic and clinical characteristics of patients with CAT in the US; and (2) understand real-world anticoagulant treatment patterns observed in the inpatient hospital or emergency department (ED) and post-discharge/outpatient settings.

\section{Methods}

\section{Study design and data sources}

This retrospective cohort study utilized three real-world databases: (1) IQVIA Hospital Charge Data Master (CDM) database, (2) IQVIA Patient Centric Pharmacy Claims Database (LRx), and (3) IQVIA Patient Centric Medical Claims Database (Dx) from January 1, 2015 to May 31, 2018.

The CDM database was used to identify anticoagulant therapies received during an inpatient or ED visit. It is comprised of data collected from hospital operational files from over 650 non-federal, acute-care short-stay hospitals. Data elements include all inpatient and outpatient encounters with detailed information on diagnoses, procedures, treatment, and patient and hospital characteristics.

The LRx database was used to identify anticoagulants received from outpatient pharmacies. It consists of pharmacy claims for dispensed prescriptions collected from pharmacies covering approximately $90 \%$ of all dispensed prescriptions from US retail pharmacies and over 1.4 billion prescriptions per year, representing claims from all payer types. The Dx database was used to identify anticoagulant treatments administered during outpatient physician office visits. It is composed of approximately 1 billion outpatient medical claims per year submitted by over 860,000 practitioners in the US.

In compliance with the Health Insurance Portability and Accountability Act (HIPAA), patient data were de-identified and, therefore, informed consent and institutional review board (IRB) review were unnecessary.

\section{Study population}

Patients with CAT were identified during an inpatient or ED visit in CDM between 7/1/2015 and 4/30/2018. Adult patients (age $\geq 18$ ) with a hospital admission or ED visit with primary discharge diagnosis of DVT or PE (an approach that has been shown to have positive predictive value for identifying VTE of 95\%; 95\% CI 93-97) [17, 18] were included. The first eligible inpatient or ED visit was defined as the index hospital visit and the admission date as the index date. Patients were linked to LRx and Dx, and had evidence of cancer during the 6-months prior to, on, or within 30 days after the index date. Evidence of cancer was defined as $(1) \geq 1$ inpatient claim with a cancer diagnosis; (2) $\geq 2$ outpatient medical claims with a diagnosis code for the same cancer type; or (3) $\geq 1$ outpatient medical claim with a cancer diagnosis and $\geq 1$ medical or pharmacy claim for cancer treatment (chemotherapy, biologic treatment, cancer-related hormone therapy, radiation, or cancer-related surgery). Diagnosis codes were identified in CDM and Dx 
using International Classification of Diseases, Ninth/Tenth Revision, Clinical Modification (ICD-9/10-CM) codes. Cancer treatments were identified in Dx and LRx using Current Procedural Terminology (CPT) codes, Healthcare Common Procedure Coding Systems (HCPCS) Level II codes, and/ or National Drug Codes (NDCs). Cancer-related surgery was captured in CDM using CPT codes. Patients with atrial fibrillation/flutter or mechanical heart value during the 6-months before or during the index hospital visit; or inferior vena cava filter or pregnancy during the study period were excluded. Patients had 6 months of data availability before the index date and $\geq 1$ month of follow-up after the discharge date (Fig. 1). Baseline characteristics were assessed during the 6-month pre-index period and on the index date. Details are provided in Table 1.

\section{Anticoagulant treatment during index hospital visit and transition following discharge}

Anticoagulants used during the index hospital visit were captured from billing descriptions in CDM. The index CAT treatment was defined as the first anticoagulant observed. Six treatment groups were formed: (1) DOACs (apixaban, edoxaban, dabigatran, rivaroxaban), (2) LMWH (dalteparin sodium, enoxaparin sodium, fondaparinux sodium, tinzaparin sodium), (3) warfarin (warfarin sodium), (4) UFH (heparin sodium), (5) thrombolytic agents (alteplase, urokinase), and (6) no anticoagulant treatment. If LMWH or UFH were observed on the same date as an oral anticoagulant, the patient was grouped into the specified oral anticoagulant subgroup (e.g., a patient with LMWH and a DOAC on the same date was classified as a DOAC patient). If LMWH and UFH were observed on the same date, the patient was grouped into the LMWH subgroup.

The first outpatient anticoagulant received within 3 months after discharge was captured from the Dx and LRx databases in patients with $\geq 3$ months of continuous followup after discharge. Sensitivity analyses were conducted in patients with $\geq 1$ and $\geq 6$ months of follow-up. Results of sensitivity analyses are available in supplemental tables and figures.

\section{Post-discharge anticoagulant treatment patterns}

Outpatient anticoagulant treatment patterns were evaluated in patients with $\geq 3$ months of follow-up after discharge and $\geq 3$ months of follow-up after initiation of the outpatient therapy. Discontinuation, persistence, and adherence were measured. Discontinuation was defined as a gap $>60$ days between the end of days' supply of a prescription and the next dispensing date of any drug in the same treatment group or as a switch to a new treatment group; discontinuation date was defined as the dispensing date + days' supply for the prescription before the gap. Persistence was defined as remaining on therapy with no gaps $>60$ days between the end of days' supply for a prescription to the next dispensing date of any drug in the same treatment group. The proportion of patients with persistence to the initial outpatient treatment of $\geq 3$ months was reported. Adherence was assessed using medication possession ratio (MPR), calculated as the sum of the days' supply of all claims occurring before the discontinuation date divided by the number of days between the treatment start date and discontinuation date, and capped at $100 \%$. Adherence was defined as MPR $\geq 80 \%$.
Fig. 1 Patient selection flow chart

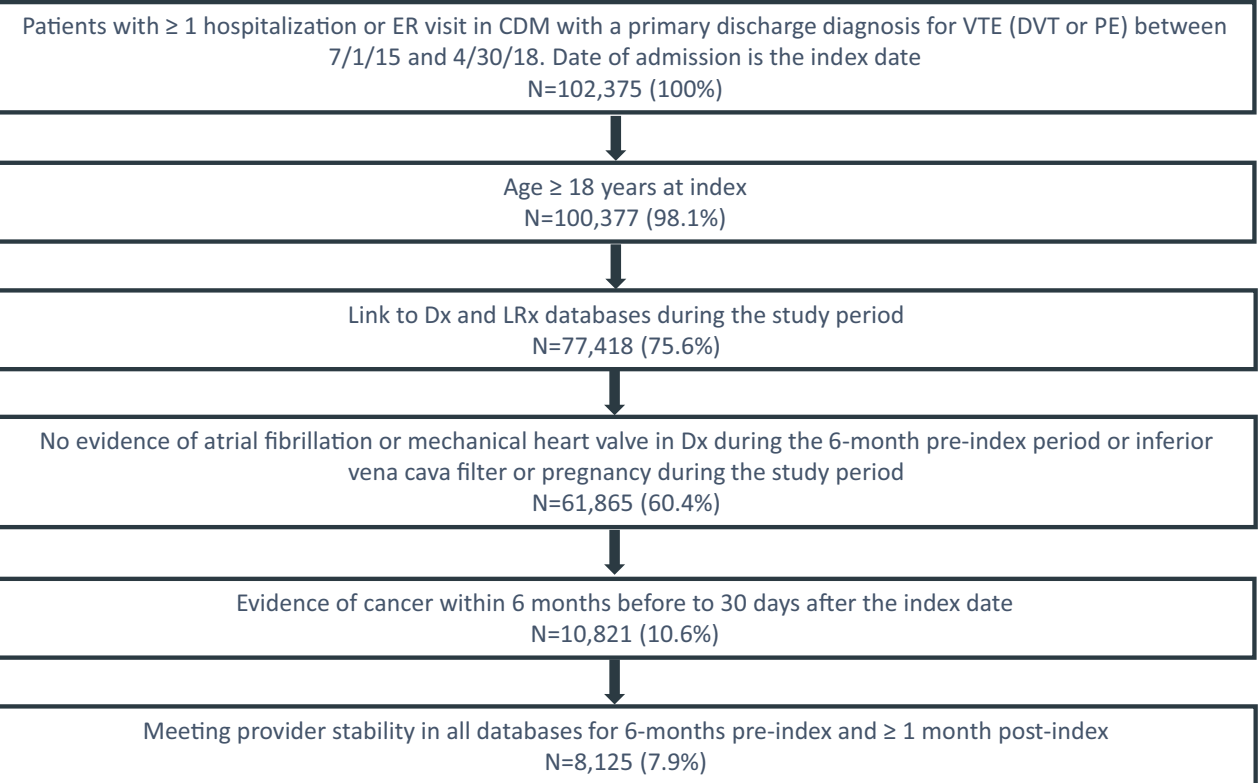




\section{Statistical analysis}

This study was descriptive in nature. Mean and standard deviation (SD) were presented as measures of central tendency and variance for continuous variables. Frequency $(\mathrm{N})$ and percentage (\%) of patients in each cohort were reported for categorical variables. All analyses were conducted using SAS version 9.4 (Cary, NC, USA).

\section{Results}

\section{Patient baseline characteristics}

In total 8125 patients with CAT identified during an inpatient or ED visit were included (Fig. 1). Mean age was 65.6 years $(\mathrm{SD}=13.0), 46.7 \%$ were male, and average length of stay of the index hospital visit was 4 days $(\mathrm{SD}=4.0)$. Use of prior CAT treatments was observed in $60.2 \%$ of patients, and $8.0 \%$ had a prior CAT diagnosis. Common comorbidities included hypertension (61.3\%) and dyslipidemia (39.7\%). Lung (18.5\%) and breast (14.5\%) cancer were the most common primary cancer types. Almost half of patients had no oncology treatment (48.6\%), and 39.5\% had chemotherapy (Table 1).

\section{Transition of CAT treatment from the inpatient/ED setting to the outpatient setting}

A total of 5341 patients (65.7\%) had $\geq 3$ months of post-discharge follow-up. LMWH and UFH were the most common initial anticoagulants during the index hospital visit (35.2\% and $27.4 \%$, respectively), followed by DOACs (9.6\%). Anticoagulant therapy was not observed in $20.8 \%$ of patients (Fig. 2).

Over $70 \%$ of patients who started treatment with DOACs $(\mathrm{n}=514)$ remained on DOACs after discharge $(71.4 \%)$. Conversely, only $24.1 \%$ of patients treated with LMWH and $43.5 \%$ treated with warfarin during the index hospital visit remained on the index CAT treatment after discharge (Fig. 2). Among patients with no anticoagulant treatment during the index hospital visit $(\mathrm{N}=1110), 46.2 \%$ received DOACs, $13.6 \%$ received LMWH, 7.1\% received warfarin, and $32.7 \%$ did not receive outpatient treatment within 3 months after discharge.

Within 3 months after discharge, DOACs were most frequently used in the outpatient setting (40.3\%), followed by LMWH (18.0\%) and warfarin (10.7\%); nearly one-third of patients (30.5\%) had no outpatient anticoagulants (Fig. 2). Treatment transition patterns from the index hospital visit to the outpatient setting were similar with 1- and 6-month follow-up periods (Supplemental Figs. 3 and 4).
Post-discharge outpatient anticoagulant treatment patterns

Among the 5341 patients with $\geq 3$ months of follow-up after discharge, a total of 2243 patients had a claim for outpatient anticoagulant therapy within 3 months after discharge, and had $\geq 3$ months of follow-up after outpatient treatment initiation (Table 2). Overall, $23.3 \%$ of patients discontinued the outpatient anticoagulant therapy within 3 months, with a lower rate of discontinuation in patients treated with DOACs (12.2\%) or warfarin (17.2\%) compared to LMWH (47.0\%) and UFH (50.0\%). A higher proportion of patients treated with DOACs and warfarin were persistent to the initial outpatient anticoagulant at 3 months after treatment initiation compared to those treated with LMWH and UFH (DOACs: 87.8\%; LMWH: 53.0\%; warfarin: 82.8\%; UFH: 50.0\%). Oral anticoagulants had higher adherence than parenteral anticoagulants; $88.8 \%$ of patients treated with DOACs and $89.0 \%$ of warfarin patients had MPR $\geq 80 \%$ compared to $76.4 \%$ of patients treated with LMWH (Table 2). Similar trends were observed in patients treated with anticoagulants within 1- and 6-months of discharge from the index hospital visit (Supplemental Tables 3 and 4).

\section{Discussion}

This study is the first publication that provides a recent look at anticoagulant treatment patterns using real-world data after the release of the 2019 NCCN guideline supporting use of DOACs as an alternative to LMWH in cancer patients [13] and promising clinical trial results showing lower rates of recurrent VTE in patients treated with DOACs compared to LMWHs [11, 12]. It examined the use of anticoagulant therapy in CAT patients during an inpatient or ED visit, and allowed for longitudinal tracking of CAT patients from the initial hospital visit to the post-discharge outpatient setting to evaluate transition of therapy between settings.

Consistent with literature [14, 15], LMWH and UFH were the most common anticoagulants used to treat CAT during an inpatient or ED visit (35.2\% and $27.4 \%$, respectively; Fig. 2). Results also suggests that, consistent with NCCN guidelines [13], DOACs are being used as first-line CAT treatment in the hospital setting. DOACs were the most commonly observed post-discharge anticoagulant treatment (46.2\%), and most patients who started DOACs in the hospital setting stayed on DOACs after discharge (71.4\%) (Fig. 2). In addition, this study observed that patients treated with outpatient oral anticoagulants stayed on their anticoagulant therapy longer and with better adherence, compared to parenteral anticoagulant therapy. The proportion of patients with persistence $\geq 3$ months after outpatient treatment initiation ranged from $82.8-87.8 \%$ for oral and $50.0-53.0 \%$ for 


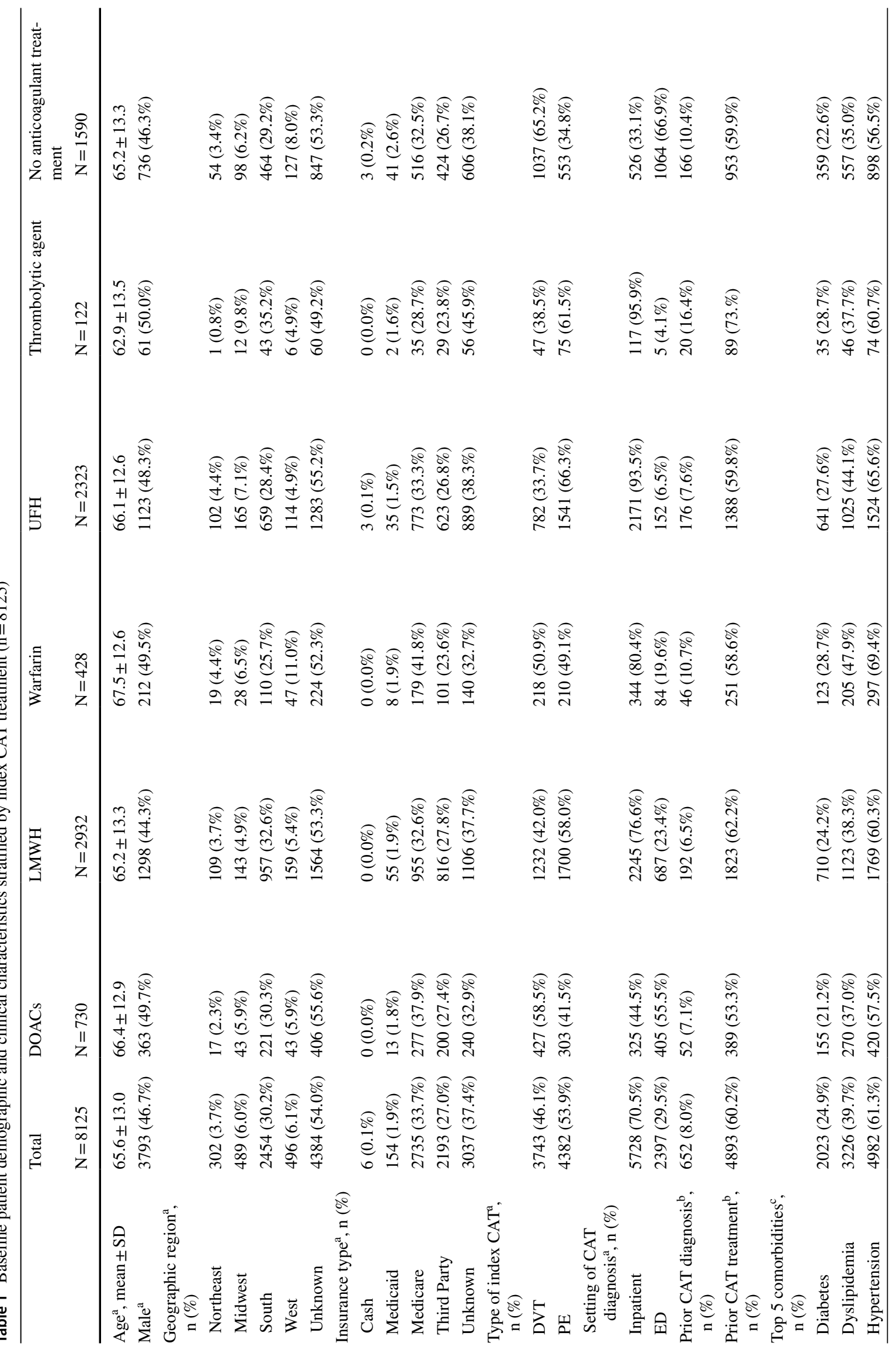




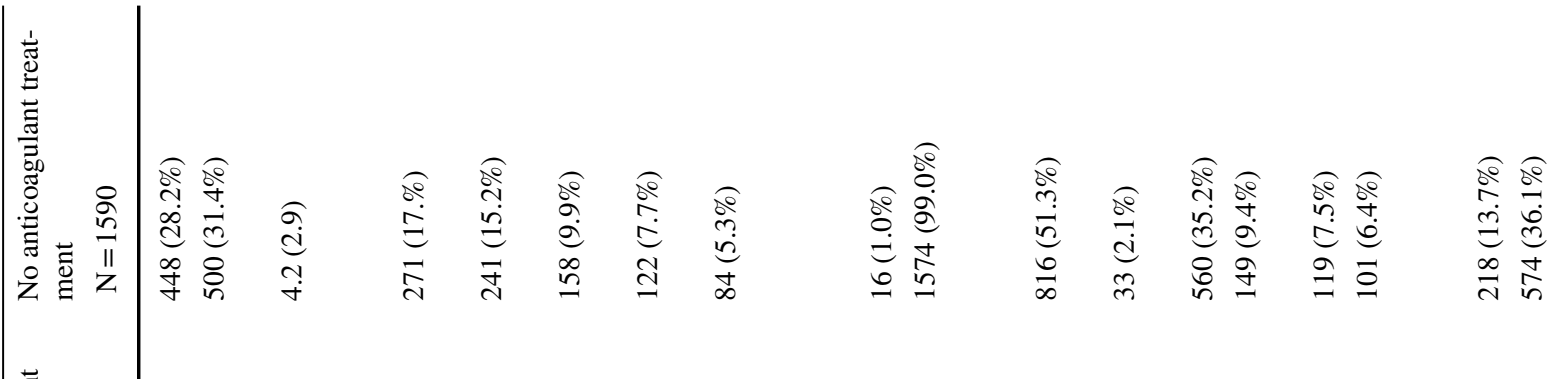

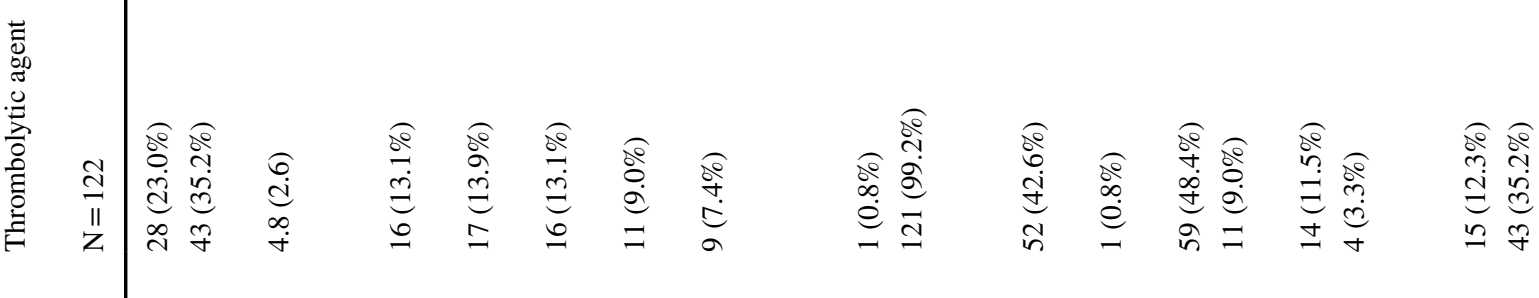

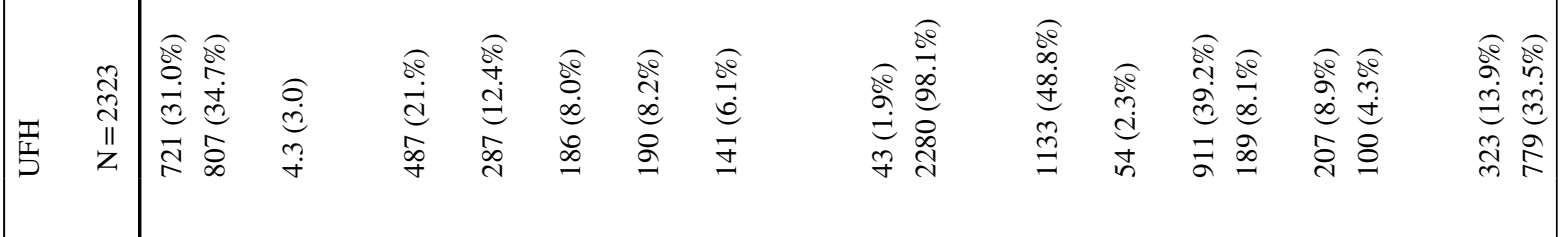

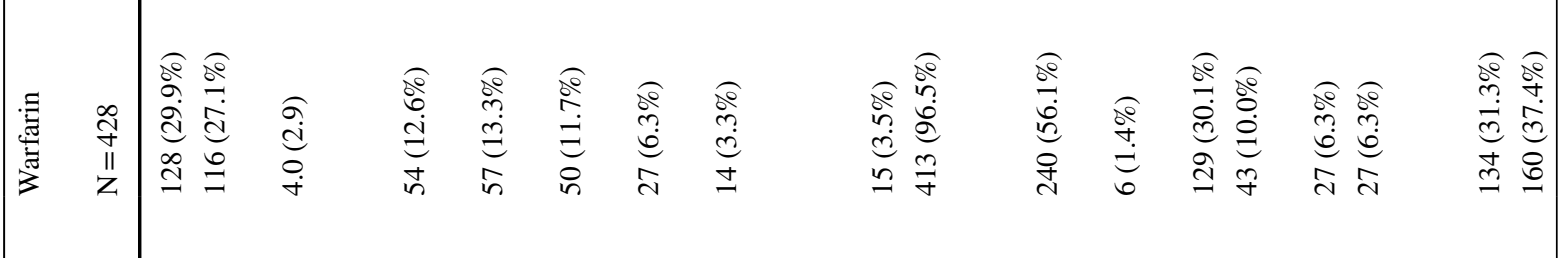

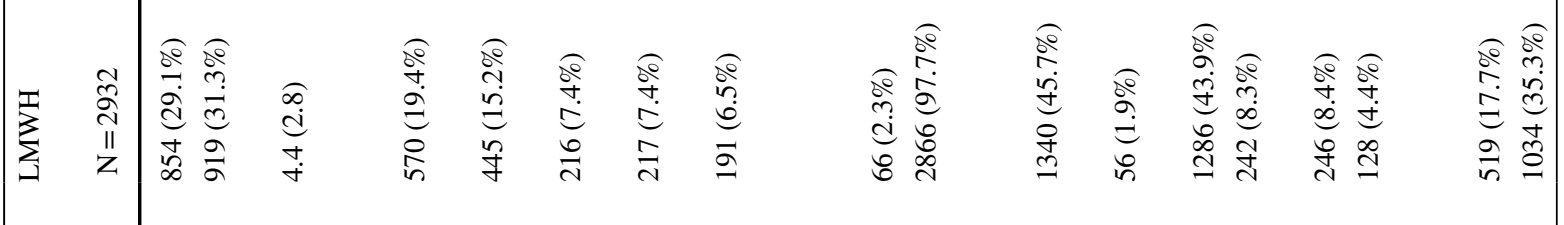

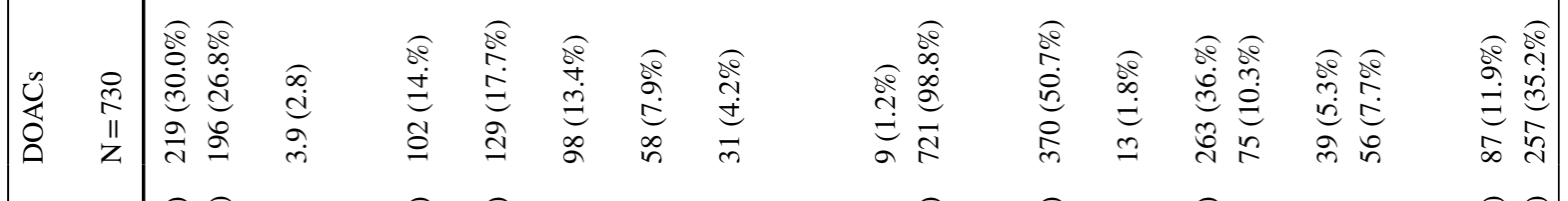

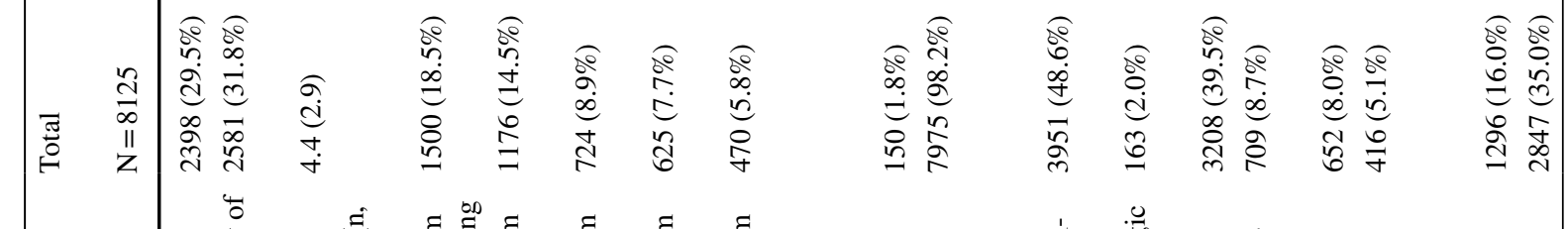

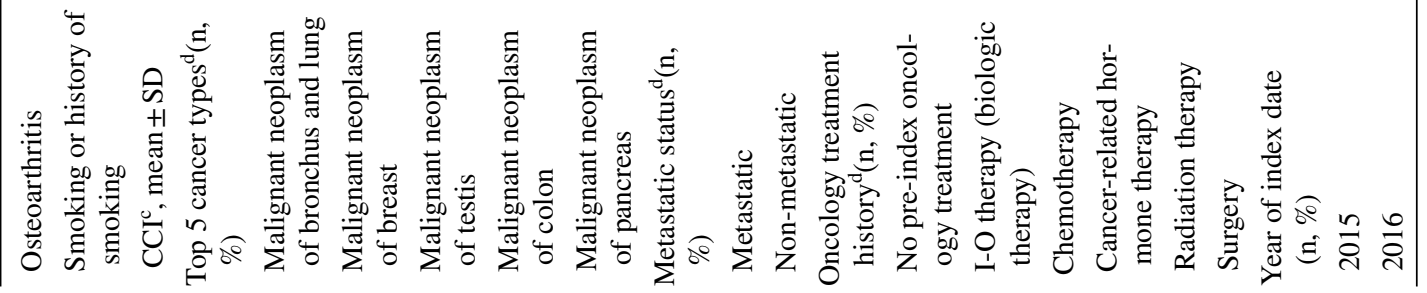




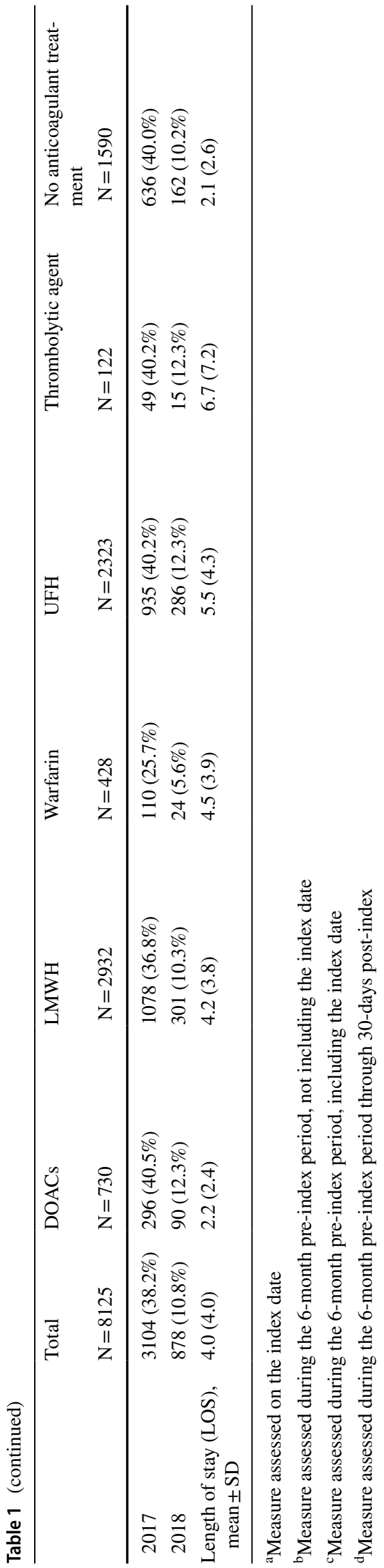

injectable anticoagulants; $89 \%$ of patients treated with oral anticoagulants and $0-76.4 \%$ of patients treated with injectable anticoagulants had high adherence (Table 2). Khorana et al. reported similar findings in their 2017 publication; patients initiating LMWH had shorter persistence and were more likely to discontinue than patients treated with DOACs or warfarin [14].

Roughly $20 \%$ of CAT patients in this study did not receive anticoagulant treatment during the index hospital visit (Fig. 2). As cancer patients may present themselves to the hospital with other complex clinical conditions, it's possible that physicians prioritized treating more urgent conditions over CAT or had other compelling clinical justification not to initiate anticoagulant therapy. Furthermore, patients may have received non-pharmacological interventions (i.e., thrombolysis) which were not assessed. Approximately $31 \%$ of patients who were treated for their CAT in the hospital did not receive further outpatient anticoagulant therapy in the 3 months following discharge (Fig. 2). This result is in line with a prior claims database study published in 2015 which found that $30 \%$ of CAT patients were not treated with anticoagulants [19]. Further research exploring reasons for not receiving CAT is warranted.

This study has several strengths. This is the first study to describe anticoagulant treatment patterns in CAT patients from the inpatient through the post-discharge outpatient setting. The use of a large hospital database allowed for a diverse cohort of patient types and hospital settings, and the ability to capture health care records for patients regardless of health plan or payer type, supporting the generalizability of the findings. Furthermore, the availability of outpatient pharmacy and medical claims allowed for an assessment of treatment changes between the inpatient/ED and outpatient settings, as well as adherence and persistence of the outpatient treatment.

As with all observational research, there are inherent limitations to this study. The data were collected for billingpurposes rather than research, and key information about treatment decisions (likely influenced by clinical risk factors), physician preference, patient preference or insurance coverage issues, are lacking. The LRx and Dx databases are open-source data and might not capture complete patient activity. Finally, clinical endpoints, such as post-discharge recurrent VTE or major bleeding adverse events, were not evaluated due to the limited sample size having sufficient follow-up.

\section{Conclusion}

While LMWH was most commonly used to treat CAT during an inpatient/ED visit, patients were also treated with DOACs in this setting, consistent with current treatment guidelines 


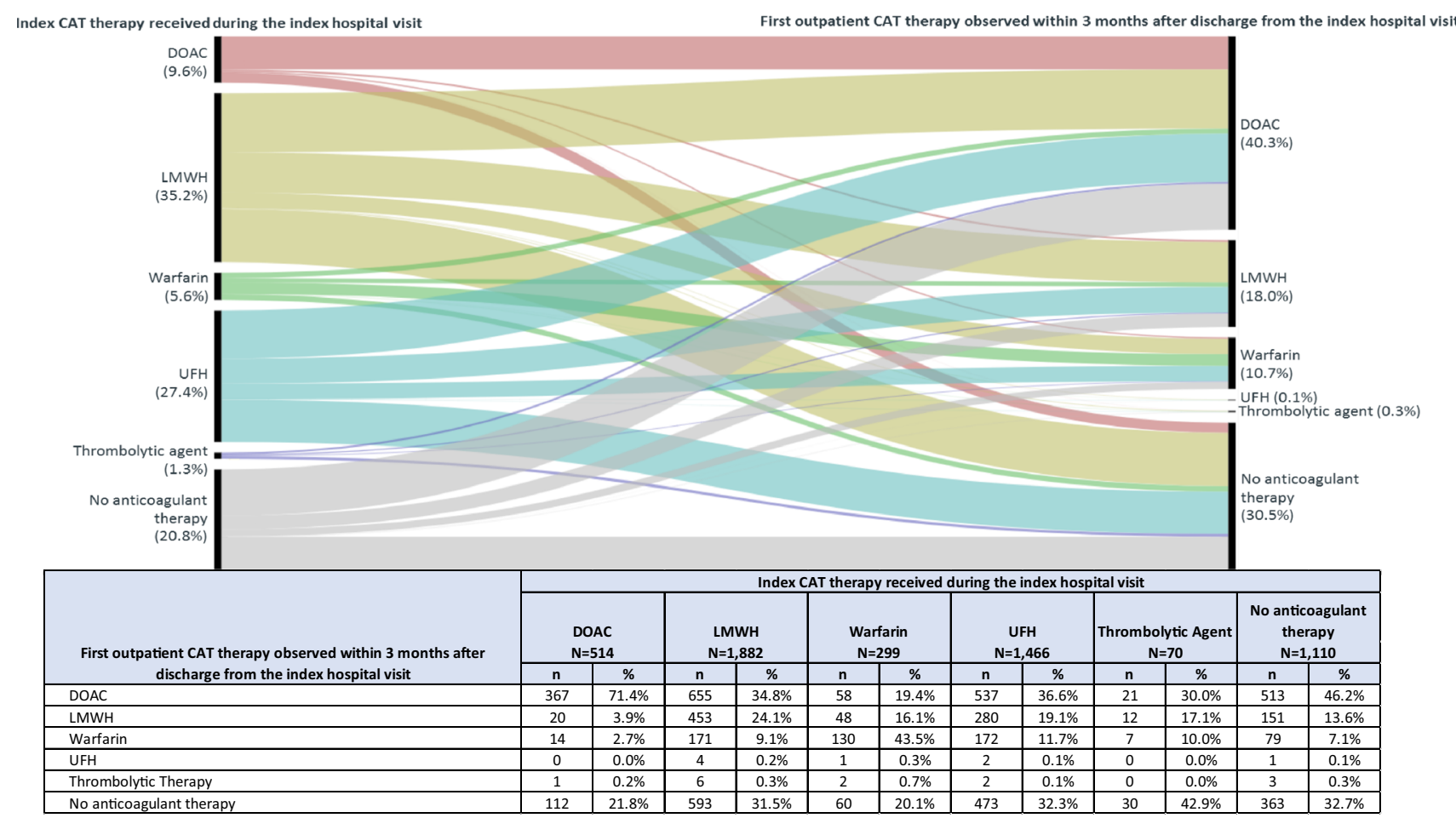

Fig. 2 Sequence of the initial anticoagulant therapies received during the index hospital visit and within 3 months after discharge in patients with at least 3 months of follow-up $(n=5341)$. Values provided are the percentage of patients who were treated with the specified therapies during the index hospital visit (left-hand bar), and the initial treatment received in the outpatient setting within 3 months after discharge (right-hand bar). The shaded pathways represent the proportion of patients who flow from the specified hospital treatment to the specified outpatient treatments

Table 2 Treatment patterns of the initial post-discharge anticoagulant treatment received within 3 months after discharge among CAT patients with $\geq 3$ months of follow-up after discharge and $\geq 3$ months of follow-up after initiation of the outpatient treatment $(n=2243)$

\begin{tabular}{|c|c|c|c|c|c|c|}
\hline & $\begin{array}{l}\text { Total } \\
\mathrm{N}=2243\end{array}$ & DOACs $N=1300$ & LMWH N $=526$ & Warfarin $\mathrm{N}=408$ & $\begin{array}{l}\text { UFH } \\
\mathrm{N}=6\end{array}$ & $\begin{array}{l}\text { Thrombolytic therapy } \\
\mathrm{N}=3\end{array}$ \\
\hline $\begin{array}{l}\text { Patients with discontinuation } \\
\text { within } 3 \text { months of treatment } \\
\text { initiation }{ }^{\mathrm{a}}(\mathrm{n}, \%)\end{array}$ & $523(23.3 \%)$ & $159(12.2 \%)$ & $247(47.0 \%)$ & $70(17.2 \%)$ & $3(50.0 \%)$ & $3(100.0 \%)$ \\
\hline $\begin{array}{l}\text { Persistence to therapyat } 3 \\
\text { months after treatment } \\
\text { initiation }{ }^{\mathrm{b}}(\mathrm{n}, \%)\end{array}$ & $1720(76.7 \%)$ & $1141(87.8 \%)$ & $279(53.0 \%)$ & $338(82.8 \%)$ & $3(50.0 \%)$ & $0(0.0 \%)$ \\
\hline $\mathrm{MPR}^{\mathrm{c}}$, mean $\pm \mathrm{SD}$ & $0.9(0.1)$ & $0.9(0.1)$ & $0.9(0.2)$ & $0.9(0.1)$ & $0.4(0.3)$ & $1.0(0.0)$ \\
\hline Adherence (MPR $\geq 0.80 ; \mathrm{n}, \%)$ & $1923(85.7 \%)$ & $1154(88.8 \%)$ & $402(76.4 \%)$ & $363(89.0 \%)$ & $0(0.0 \%)$ & $3(100.0 \%)$ \\
\hline
\end{tabular}

1631 patients did not have evidence of anticoagulant treatment within 3 months after discharge; 1467 patients had outpatient anticoagulant therapy, but had less than 3 months of follow-up after outpatient treatment initiation.

${ }^{a}$ Discontinuation is defined as a gap of $>60$ days between end of days' supply for a prescription to the next dispensing date of a drug in the same treatment group, or as a switch to a new treatment group. Last date of days' supply before this gap is the discontinuation date

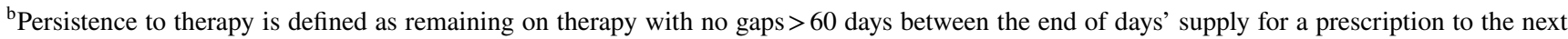
fill date of any drug in the same treatment group

${ }^{c}$ MPR is defined as the sum of days' supply for all claims prior to the discontinuation date (i.e., while a patient is on therapy)

and prior recommendations. Furthermore, patients treated with DOACs in the hospital were less likely to switch to a different therapy after discharge. Patients treated with oral anticoagulants post-discharge in the outpatient setting had better persistence after treatment initiation and better adherence than patients treated with parenteral anticoagulants. This study adds evidence supporting the use of DOACs to treat CAT. Future real-world studies to evaluate effectiveness 
and safety of DOACs with longer follow-up and larger sample size are warranted to further inform the complex clinical decisions in patients with CAT.

Acknowledgements The authors would like to thank Jin Wu for providing statistical expertise and programming.

\section{Compliance with ethical standards}

Conflict of interest Guo JD, Wygant G, Pan X, Rajpura J, Stwalley B, and Rosenblatt L are employees of Bristol-Myers Squibb which provided funding for this study. Lane D was an employee of Bristol-Myers Squibb at the time this study was conducted. Poretta $T$ is an employee of Rutgers University and a consultant of Bristol-Myers Squibb. Hlavacek $\mathrm{P}$ is an employee of Pfizer, Inc. Gorritz $\mathrm{M}$, Wang X, Chen CC, and Wade RL are employees of IQVIA, Inc, which received funding from Bristol-Myers Squibb to conduct this study.

Open Access This article is licensed under a Creative Commons Attribution 4.0 International License, which permits use, sharing, adaptation, distribution and reproduction in any medium or format, as long as you give appropriate credit to the original author(s) and the source, provide a link to the Creative Commons licence, and indicate if changes were made. The images or other third party material in this article are included in the article's Creative Commons licence, unless indicated otherwise in a credit line to the material. If material is not included in the article's Creative Commons licence and your intended use is not permitted by statutory regulation or exceeds the permitted use, you will need to obtain permission directly from the copyright holder. To view a copy of this licence, visit http://creativecommons.org/licenses/by/4.0/.

\section{References}

1. Kahale L, Hakoum M, Tsolakian I et al (2018) Anticoagulation for the long-term treatment of venous thromboembolism in people with cancer. Cochrane Database Syst Rev 6:CD006650

2. Timp J, Braekkan S, Versteeg H, Cannegieter S (2013) Epidemiology of cancer-associated venous thrombosis. Blood 122(10): 1712-1723

3. American Heart Association. What is venous thromboembolism? http://www.heart.org/en/health-topics/venous-thromboembolism /what-is-venous-thromboembolism-vte. Accessed 12 Feb 2019

4. Sheth R, Niekamp A, Quencer K et al (2017) Thrombosis in cancer patients: etiology, incidence, and management. Cardiovasc Diagn Ther 7(Supple 3):S178-S185

5. Berger J, Seheult R, Laliberte F et al (2018) Clinical outcomes of prolonged anticoagulation with rivaroxaban after unprovoked venous thromboembolism. Res Pract Thromb Haemast 2:58-68

6. Kraaijpoel N, Carrier M (2019) How I treat cancer-associated venous thromboembolism. Blood 133(4):291-298
7. Cohen AT, Katholing A, Rietbrock S, Bamber L, Martinez C (2017) Epidemiology of first and recurrent venous thromboembolism in patients with active cancer. A population-based cohort study. Thromb Haemost 117(1):57-65

8. Razak N, Jones G, Bhandari M, Berndt M, Metharom (2018) Cancer-associated thrombosis: an overview of mechanisms, risk factors and treatment. Cancers 10:1-21

9. Khorana AA et al (2008) Development and validation of a predictive model for chemotherapy-associated thrombosis. Blood 111:4902-4907

10. Prandoni P, Lensing AW, Piccioli A et al (2002) Recurrent venous thromboembolism and bleeding complications during anticoagulant treatment in patients with cancer and venous thrombosis. Blood 100(10):3484-3488

11. McBane RD, Wysokinski WE, Le-Rademacher J et al (2018) Apixaban, dalteparin, in active cancer associated venous thromboembolism, the ADAM VTE trial. Blood 132:421

12. Young AM, Marchall A, Thirlwall J et al (2018) Comparison of an oral factor Xa inhibitor with low molecular weight heparin in patients with cancer with venous thromboembolism: results of a randomized trial (SELECT-D). J Clin Oncol 36(20):2017-2023

13. National Comprehensive Cancer Network. NCCN Clinical Practice Guidelines in Oncology. Cancer-associated venous thromboembolic disease. Version 1.2019. February 28, 2019

14. Khorana A, McCrae K, Milentijevic D et al (2017) Current practice patterns and patient persistence with anticoagulant treatments for cancer-associated thrombosis. Res Pract Thromb Haemast $1: 14-22$

15. Xiang E, Ahuja T, Raco V et al (2018) Anticoagulation prescribing patterns in patients with cancer. $\mathrm{J}$ Thromb Thrombolysis 45(1):89-98

16. Guo JD, Hlavacek P, Poretta $T$ et al Treatment patterns in patients with cancer-associated venous thromboembolism in the US: a real world retrospective database analysis. In: Proceedings of the American Associated for Cancer Research Annual Meeting 2019; 2019 Mar 20-Apr 3; Atlanta, GA. Philedelphia (PA): AACR; Cancer Res 2019;79(13 Suppl):Abstract nr 2267

17. White RH, Garcia M, Sadeghi B et al (2010) Evaluation of the predictive value of ICD-9-CM coded administrative data for venous thromboembolism in the United States. Thromb Res 126:61-67

18. Fang MC, Fan D, Sung SH et al (2017) Validity of using inpatient and outpatient administrative codes to identify acute venous thromboembolism: the CVRN VTE study. Med Care 55:e137-e143

19. Masserie C, Kariburyo MF, Mardekian J et al (2015) Clinical outcomes and treatment patterns of venous thromboembolism among cancer patients in a large commercial database. Value Health 18:A1-A307

Publisher's Note Springer Nature remains neutral with regard to jurisdictional claims in published maps and institutional affiliations. 